Chapter 8

\title{
Use of Self-Assembly Nanofibre Biomaterials for Neural Repair After Injury
}

\author{
Mingyong Gao, Jiasong Guo, \\ Gilberto K. K. Leung and Wutian Wu \\ Additional information is available at the end of the chapter \\ http://dx.doi.org/10.5772/57098
}

\section{Introduction}

\subsection{Central nervous system injury}

Traumatic brain injury (TBI) and spinal cord injury (SCI) are serious health problems in society. It is estimated that approximately 1.7 million TBI (Ghajar, 2000) and 12,000 new cases of SCI (https://www.nscisc.uab.edu, 2011) occur each year in the U.S. TBI is the leading cause of death and permanent severe neurological disabilities in individuals aged below 45 years in the western world. Similarly, SCI affects young adults with an average age of 40.7 years, and is predominantly caused by motor vehicle accidents. Both types of central nervous system (CNS) injuries commonly result in significant sensorimotor deficits as well as psychological and cognitive impairments. The associated social-economic burden is significant.

\subsection{Peripheral nervous system injury}

Peripheral nervous injuries (PNI) are most primarily caused by traffic accident, bone fractures and joint dislocations (Millesi et al., 1998). Additionally, complications of regional anesthesia and some neuropathic or metabolic disorders may also cause PNI. The incidence is around $2.8 \%$ of trauma patients per year. Injuries to the peripheral nerves may lead to partial or complete loss of sensory, motor or autonomic functions that can seriously compromise the life quality of the patients and result in significant socioeconomic loss (Noble et al., 1998; Taylor et al., 2008). 


\section{3. current neural tissue engineering}

\subsubsection{Tissue engineering for the repair of central nerve injury}

CNS injuries are characterized by the permanent loss of neural tissues as the result of apoptosis, axonal damage, as well as acute and chronic neural degeneration. These primary and secondary neuropathological cascades lead to severe destruction of neuronal circuitry. Subsequent significant astrogliosis may also constitute a microenvironment that is inhibitory to regeneration. The capacity for self-repair within the adult CNS after injury is poor, and numerous reparative strategies have been developed to enhance axonal regrowth, reactivate the plasticity of the spared neural tissue, and replace lost tissue by means of cell transplantation (Kim et al., 2012). Of these, neural bioengineering adopts a multifaceted approach in providing both a permissive microenvironment and a suitable three-dimensional scaffold that integrates transplantable cells with bioactive factors. By modifying the components, morphology, and architecture of biodegradable and biocompatible materials, a number of scaffolds have been developed to tailor to the desired physical and chemical properties required for neural repair. Both natural and artificial polymers such as collagen, chitosan, PLGA (poly lactic-co-glycolic acid ), and nanofiberous scaffold have been tested in TBI and SCI models (Peter et al.,2009; Wang et al.,2011). When combined with engrafted stem cells and surface modification, these bioengineered scaffolds represent some of the most promising materials in neuro-regenerative therapy. A wide range of transplantable cells have been used in combination with these scaffolds, including embryonic stem cells, neural stem cells, mesenchymal stem cells, Schwann cells and a variety of adult multipotent stem cell types. The underlying principle is that these engrafted cells may promote neural repair and regeneration by means of growth factor production, neuronal replacement and remyelination. Similarly, enhancement with integrated bioactive factor or oligopeptide motifs may provide a more conducive environment for the survival of the engrafted cells and their integration with the host tissue-scaffold. More importantly, novel controllable release techniques can potentially facilitate the delivery of embedded tissue factors that counteract or neutralize the local inhibitory signals, and degrade glioscar. Given the complexity in anatomical organization and functional communication within the CNS, bioengineered scaffold-based is an reparative strategy of significant potential.

\subsubsection{Tissue engineering for repair of peripheral nerve injury}

When compared with the CNS, the PNS has a much greater capacity for regeneration after traumatic injury. For lesion gaps of over $5 \mathrm{~cm}$ in length, autologous nerve grafting is a treatment of choice. However, the recovery of sensory and motor function is often slow and incomplete. Nerve grafting is limited by the availability of the donor nerve, the loss of donor nerve function, and the additional surgical trauma and complications (Gordon et al., 2003). Peripheral nerve is mainly constituted by fascicles of myelinated and unmyelinated nerve fibers as well as multiple layers of connective tissue and blood vessels. PNS bioengineering approaches therefore require longitudinally orientated conduits to provide the physical support and contact guidance for neurite regrowth, while maintaining the biological and functional viability of the distal denervated targets during the regenerative process. The ultimate goal of 
PNS bioengineering is to develop bioengineered nerve implant that could match or exceed the performance of autograft. Currently, various conduits made of diverse synthetic or natural biomaterials have been exploited to bridge experimental nerve transection gaps of between $10 \mathrm{~mm}$ and $80 \mathrm{~mm}$ in experimental rodent and primate models. The most commonly used biomaterials are biodegradable polymers such as PLGA, type I Collagen and chitosan. The engrafted cells may include Schwann cells, neural stem cells and olfactory ensheathing cells (OECs). However, the degree of axonal regeneration and functional recovery has so far been found to be limited, and inferior to nerve grafting control. Further studies are required to explore the applications of other novel materials. (Battiston et al., 2009).

\subsection{Biomaterials developed in the field of Neural Tissue Engineering}

Various promising biomaterials have been exploited to meet the diverse needs for specific bioengineering applications. The fundamental requirements of biomaterials utilized in neural tissue engineering include biodegradability, neural bioactivity and neural tissue-matched mechanical module.

\subsubsection{Biological biomaterials}

These are mainly natural polymers such as collagen, laminin, fibronection, fibin, hyaluronic acid. agarose, alginate, and chitosan. The majority of them are derived directly from ECM and have been extensively studied due to their inherent merits including the presentation of biological receptor-binding ligands, the susceptibility to proteolytic degradation and remodeling in vivo (Ma et al., 2008). These natural macromolecules can be hydrated, and serve as bioscaffolds for various cells in vivo and in vitro. For example, collagen is the most abundant protein from natural ECM in connective tissue. In mammalian tissues, the primary structural collagen is type I collagen. The collagen conduit made of type 1 collagen such as NeuraGen conduit has been approved by the United States Food and Drug Administration (FDA) and is commercially available for clinical use practice, whereas limited in field of the peripheral nerve repair (Kehoe et al., 2012). Major concerns regarding the clinical application of biologically derived materials, include the problems with sustainable production, immunogenicity, and pathogen transmission as well as weak mechanical in vivo strength.

\subsubsection{Artificial biomaterials}

Compared to the natural polymers, artificial biomaterials or biomimetic materials, could be designed and synthesized to mimic one or multiple desired characteristics of the natural ECM for specific purposes. For reparative applications, artificial polymers have the advantages of having great flexibility for design and modification so as to allow for the control of orientation and development of new-born tissue for better functional outcomes.

\subsubsection{Degradable materials}

Biodegradability is an important property of biomaterials in tissue engineering. Due to the well-accepted biodegradability and biocompatibility, linear aliphatic polyesters including 
poly(lactic acid) (PLA), poly(glycolic acid) (PGA), and their copolymers poly(lactic acid-coglycolic acid) (PLGA) have been widely utilized to reconstruct bioscaffold in diverse conditions of neural repair. To facilitate host-material integration, biomaterial candidates must also possess the appropriate elastic module. For instance, hydrogel made of poly(2-hydroxyethyl methacrylate-co-methyl methacrylate) (p(HEMA-co-MMA)) has similar mechanical properties to the mammalian spinal cord (elastic modulus of 200 to $600 \mathrm{kPa}$ ), and could be customized by altering the ratio of co-monomers (Dalton et al.,2002). The hydrophobic surface properties in most of the synthetic biodegradable materials such as PLGA, PCL and PHB, may be modified by coating them with ECM proteins components like laminin, fibronectin, collagen. Specific adhesion oligopeptide such as RGD and IKVAV, YIGSR may also be added to improve their adhesion properties for seeded cells. Several studies showed that the cellular adhesion performance of these artificial biomaterials including methyl cellulose, alginate, poly (hydroxyethyl methacrylate) (PHEMA), poly (hydroxybutyrate) (PHB) (Samadikuchaksaraei, 2007), could be significantly improved by surface modifications. Another category of synthetic biomaterial is related to the nanofibre scaffold which will be discussed in later sections.

\subsubsection{Nondegradable materials}

The use of synthetic nondegradable materials in neural repair is limited by their nondegradablilty and unbioabsorbility. The majority of reported studies involved PNI. For example, poly (2-hydroxyethyl methacrylate) has been demonstrated to support regeneration of injured axons in a rat SCI model (Tsai et al. 2006). Recently, electroactive polymer like polypyrole or hybrid conduct materials showed neuronal attachment and growth. The major concerns regarding these materials are related to immunorejection, chronic inflammatory responses, fibrous scarring, and the associated problems of neural compression and need for re-operation. These render nondegradable materials unsuitable for CNS repair (Cullen et al., 2008).

\section{Nanofibrous scaffolds applied in CNS regeneration}

\subsection{Current fabrication of nanofiber-based bioscaffolds used in CNS regeneration}

The aim of biomedical engineering is the design and development of novel biomaterials that can recapitulate the key characteristics of natural ECM with the associated topographical cues, cellular adhesion sites, biochemical signals and physiological viscoelastic modules. In general, extracellular proteins such as collagen, fibrin and glycosaminoglycans, possess fibrous structures with diameters on the nanometer or sub-micrometer scales. Several bioengineering approaches have been developed for the fabrication of artificial nanofibre constructs with diameters that range from 10 to $100 \mathrm{~nm}$.

\subsubsection{Self-assembling peptide nanofiber scaffolds (SAPNS)}

Many biological macromolecules such as like phospholipids can readily self-assemble to form highly ordered bio-structures through van der Waals forces, hydrogen bonds, ionic bonds, and 
hydrophobic interactions. To recapitulate the bioactive motif of laminin, Stupp and coworkers designed a self-assembling peptide amphiphile (PA) that consisted of the laminin-derived peptide IKVAV (Ile-Lys-Val-Ala-Val). The self-assembly of IKVAV could be initiated spontaneously upon the introduction of physiological buffer which leads to the formation of nanofibers with diameter ranging from 6 to $8 \mathrm{~nm}$ (Tysseling-Mattiace, 2008).

\subsubsection{Eletrospun nanofiber scaffolds}

Electrospinning was a traditional industrial fabrication technique used widely in 1930s. Due to its effectiveness of producing microfibers with diameters of sub-micron or nanometer scale, it has been utilized to process a number of natural and synthetic polymers such as collagen, fibroin, PLLA, PLGA, and PCL. The electrospun nanofiber matrices resemble the structural morphology of ECM with a high surface area-to-volume ratio, which has been shown to greatly facilitate cellular attachment, proliferation and differentiation. Recent studies continue to report the development of novel electrospun nanofibers, and the introduction of bioactive molecules such as growth factors during the fabrication process of nanofibers for peripheral nerve regeneration (Prabhakaran et al., 2008). The intrinsic limitations of this technique include the degradation of bioactive factors during procedure, the inability to fabricate complex 3D structures or specific microstructure with designed internal pore size.

\subsubsection{Phase separation}

Phase separation, or thermally induced liquid-liquid phase separation, was developed by Ma and Zhang to produce a nanofibrous foam materials. Polymer scaffolds generated by phase separation normally have a sponge-like porous morphology with spherical pores $50-500 \mathrm{~nm}$ in diameter. Phase separation system consists of a polymer-rich component and a polymerlean/solvent-rich component, by which the polymer morphology can solidify by quenching under low temperature. A few nanofibre scaffold from artificial biodegradable polymers have been produced with phase separation including PLLA, which was studied as a suitable matrices in which NSCs can grow and differentiate (Yang et al., 2004). Compared with other techniques previously discussed phase separation has simpler and the need for specialized equipment is minimal. However, due to the small number of candidate polymers suitable for in vivo study, reports on nanofiber scaffold generated by phase separation in neural tissue engineering are limited.

\subsection{Current application of SAPNS for the repair of injured CNS}

\subsubsection{Traumatic brain injury}

The applications of RADA16-I, a representative SAPNS, in 3D cell culturing, wound hemostasis and healing has been well described in a series of studies. The initial research of RADA16I in experimental TBI was conducted by Ellis-Behnke et al. (Ellis-Behnke et al. 2006). Using an acute TBI model in which the midbrain of P2 hamsters was injured surgically with a knife wound (1.5 mm deep and $2.0 \mathrm{~mm}$ wide), $10 \mu \mathrm{l}$ of $1 \%$ SAPNS was applied to bridge the injury gap. The central traumatic lesion showed restoration in all SAPNS-treated animal subjects 
within the first 24-hour and at all other timepoints up to 30 days post-injury. Compared with saline-treatment, SAPNS created a seamless connection across lesion site and appropriate hostscaffold interfaces which led to significantly improved repair. Further studies were conducted by Guo et al. using a rodent TBI model (Guo et al., 2009). Immediately after the infliction of severe mechanical injury to the sensory-motor cortex, $20 \mu \mathrm{L}$ of $1 \%$ RADA16-I SAPNS was implanted at the lesion sites to bridge the injury gap. Histological, immunohistochemical and apoptosis studies were performed at 2 days, 2 weeks, and 6 weeks after injury. The SAPNStreated lesion sites had no cyst formation after injury and showed integrated host-scaffold interfaces; saline-treatment resulted in significant cyst formation. Moreover, SAPNS significantly reduced apoptosis in the perilesional area and effectively mitigated reactive gliosis and inflammation. Currently, a few integrative strategies of SAPNS incorporated with bioactive factors have been conducted with an aim to improve functional recovery after severe TBI.

\subsubsection{Spinal cord injury}

A variety of biodegradable hydrogel have been extensively studied in the treatment of experimental SCI. IKVAV peptide amphiphile, which consists of neuroactive pentapeptide epitope from laminin, has been applied in a moderate spinal cord contusion model in which $1 \%$ aqueous solution of IKVAV-SAPNS was injected into the lesion sites $24 \mathrm{~h}$ after injury. IKVAV-SAPNS significantly reduced the degree of oligodendroglial apoptosis perilesion and enhanced their survival rate with cleaved caspase-3 immunohistochemistry at $10 \mathrm{~d}$ after SCI. Additionally, astrogliosis was reduced significantly and the regeneration of motor-sensory axons were improved remarkably on BDA-labeling 11 week after treatment. Moreover, at 9wks after treatment, the mean locomotor score of IKVAV-SAPNS group was significantly better than that of the control group on BBB score measurement, and dorsal stepping was observed with IKVAV-treatment, indicating functional return in hindlimb movement (TysselingMattiace, 2008).

One of the most important advantages of SAPNS is its ability to provide a 3-D matrice in which neural cells can survive and differentiate. Guo and coworkers incorporated neural stem cells (NSCs) and Schwann cells in SAPNS, and transplanted them into dorsal column lesion of the cervical spinal cord. At 6 wks after implantation, there was excellent integration between the implant and the host tissue. Moreover, extensive axonal regrowth was observed with immunohistochemistry staining with NF, 5-HT, and CGRP (Guo et al., 2007). Recent reports highlighted the further applications of controlled release of bioactive factors incorporated into the SAPNS in vitro and in vivo. For instance, CT04, a cell permeable RhoA inhibitor, was incorporated into RADA16-I-SAPNS and implanted in a complete transection lesion at T9 level of the spinal cord. This novel integrative SAPNS not only reconstructed the injured nerve gap, but also elicited significant axonal regeneration and motor functional recovery. Additionally it also effectively reduced the infiltration and apoptosis of activated macrophages within the injured spinal cord. The SAPNS-based delivery of RhoA inhibitor is a potentially effective therapeutic strategy by reknitting lesion gap, attenuating secondary injury and improving axonal regeneration (Fig.1). Moreover, Gelain and Zhang's group has developed functionalized SAPNS that can improve the engraftment and neural differentiation of seeding neural 
progenitor cells in vitro, and enhance neural regeneration in vivo. With the aid of phage display technology, a functionalized SAPNS was found to demonstrate high in vitro stem cell viability and neural differentiation, as well as significantly promoted axonal regrowth and locomotor functional recovery in acute spinal cord injury (Gelain et al., 2006; 2012).

Collectively, these in vivo studies with various SCI models evidenced the significant potential of SAPNS in the repair of SCI from different aspects that SAPNS provide. Based on the current advances of fabrication and biochemistry techniques, future directions would consider introduction of updated topographical cues and more bioactive motifs or growth factors into the scaffold design to induce more robust and organized regeneration for injured central neural system, achieving more significant functional recovery.

\section{Nanofibrous scaffolds applied in PNS repair}

\subsection{Development of nanofiber biomaterials used for PNS regeneration}

A variety of biodegradable materials have been processed into nanofibrous scaffold using eletrospining technique for PNI repair (Xie et al., 2010). In an early study, a bilayer chitosan conduit with inner layer of nano/microfibrous structure modified with oligopeptide was generated to repair a $15 \mathrm{~mm}$ sciatic nerve gap in rats (Wang et al., 2008). This novel integrative chitosan conduit effectively promoted the axonal regeneration that was comparable to that of autologous nerve grafting? on histological assessment. Recently, a blend of biodegradable polymers PLGA/PCL was used to produce electrospun tubes to bridge a $10 \mathrm{~mm}$ long sciatic nerve lesion gap in rat. Four months after surgery, most of the electrospin conduit-treated animals showed neural regeneration and functional restoration on immunohistochemial studies and electrophysiological assessment (Panseri et al., 2008). More interestingly, a novel bi-layer nanofibrous nerve conduit made of poly (Llactide-co-caprolactone) and poly(propylene glycol) has been fabricated with electrospinning technique for PNI repair. The electrospin nerve conduit was designed as the luminal layer composed of longitudinally aligned nanofibers to promote axon regeneration, while the outer layer was equipped with random- organized nanofibers for mechanical support. After being implanted to bridge a $10 \mathrm{~mm}$ gap of sciatic nerve, the nanofibrous nerve conduit significantly improved the regeneration of injured peripheral axons and motor functional recovery at 2 and 12 month post-surgery (Zhu et al., 2011). More recently, the effects of fibre diameter of electrospun conduits on peripheral nerve regeneration was analyzed with a $15 \mathrm{~mm}$ sciatic nerve injury model. These fibrous conduits consisted of aligned electrospun poly ( $\varepsilon$-caprolactone) (PCL) nanofibers $(251 \pm 32 \mathrm{~nm})$ and microfibers $(981 \pm 83 \mathrm{~nm})$. The nanofiber-treated group showed significantly greater total number of myelinated fibers and thicker myelin sheaths when compared with groups that received Microfiber and Film conduits at 3 month post-treatment. The number of regenerated dorsal root ganglion neurons in animals that received nanofiber conduits was increased significantly by retrograde labeling with fluorogold. On electrophysiological testing including compound 
muscle action potential and distal motor latency, the nanofibre-group showed greater improvement than the microfiber group (Jiang et al., 2012). These positive observations provide useful insights for the applications of electrospun nanofibrous nerve conduits with designed nanostructure in the development of peripheral nerve guide conduits.

\subsection{Reconstruction of injured PNS with SAPNS}

SAPNS has a well-defined sequence of L-amino acids that self-assemble under physiological conditions to form a fibrous scaffold within the nanoscale ( $\sim 10 \mathrm{~nm}$ in diameter). In the recent decades, SAPNS has been shown to facilitate the survival and growth of various neural cells within a 3D matrice, and effectively improved the axonal regeneration and tissue repair in context of CNS injuries. It also has significant potential for PNI repair. For example, a novel SAPNS-based nerve conduit was generated by RAD-I SAPNS ensheathed with a segment of aortic wall. With a sciatic nerve transection model of rat, the SAPNSnerve conduit was used to bridge a $10 \mathrm{~mm}$ nerve gap. Neural histomorphology, retrogradelabeling and locomotor functional assessments demonstrated significant therapeutic effects of SAPNS-based nanofiber conduit implant on axonal regeneration, remyelination and target reinnervation (Fig.2,). Additionally, SAPNS acts as a designer peptide backbone and provides the opportunity to integrate various growth factor, or functional motifs for cell adhesion, differentiation and homing, and to define and direct biological commitment of seeding cells; furthermore, functional SAPNS will provide better support for viability, migration and differentiation of engrafted stem cells in vivo and lead to better performance of neural repair in vivo.

\section{Conclusion}

The functional repair of peripheral and central nervous system injuries is a major challenge. Based on the advances of development of novel biomaterials, biochemistry and fabrication techniques, bioengineered scaffold enhanced with bioactive motifs and engrafted cells can provide a regeneration-facilitating environment for injured nervous tissues, and effectively promote the host's capacity of neural regeneration and plasticity. Numerous in vitro models have demonstrated that 3-D bioscaffold, in particular the nanofibre scaffold, can greatly support the attachment, proliferation, migration and neural differentiation of various neural cells. Moreover, significant neuroprotection and axonal regeneration have been achieved in in vivo neural injury models after treatment with these novel scaffolds. More importantly, the recovery of injured sensory and locomotor function has been shown to occur in a number of peripheral and central injury models using different animal species. However, critical issues such as functional integration of host-implant, organized regeneration pattern with updated bioengineering scaffolds and further restoration of useful neurology function, remain to be addressed in future researches.

Immunohistochemistry with Neurofilament(NF) staining showed a number of NF-positive axons (Green)penetrated into the lesion sites with SAPNS+CT04 treatment(A). Counterstained 
DAPI area (blue) indicated the gross structure of the injured spinal cord. (B) is the high power magnification corresponding to the boxed area of (A) demonstrated the NF-positive axons in the center of lesion area. Quantification analysis of the NF-positive axons in the center of the lesion area indicated that axonal regeneration was significantly improved by the SAPNS+CT04 implants compared to the only SAPNS group (Student $t$ test, ${ }^{*} \mathrm{p}<0.05$ ).
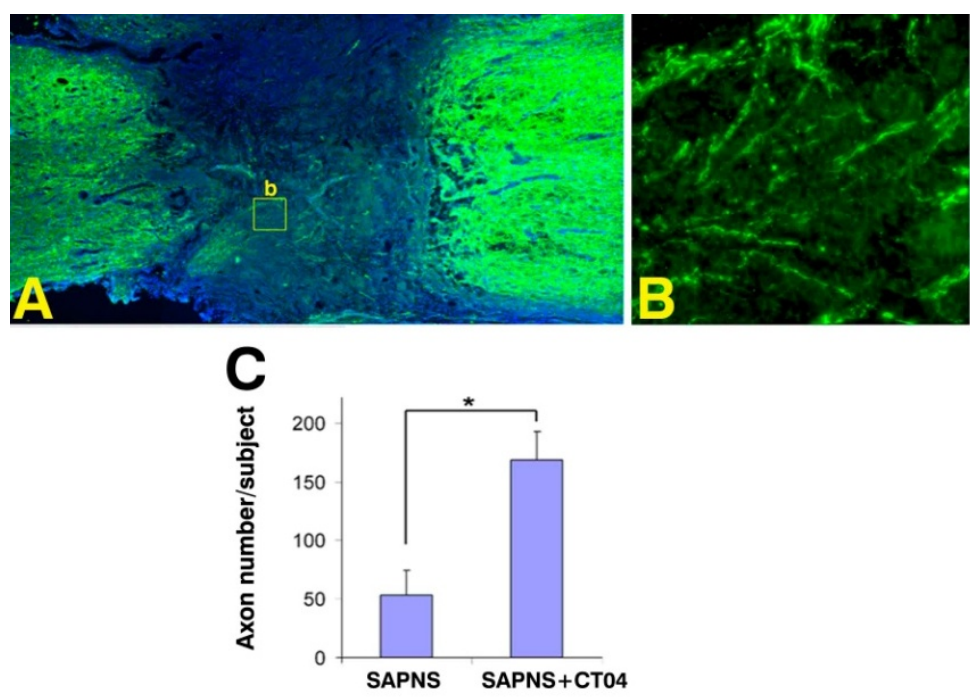

Figure 1. Axonal regeneration in the SAPNS-treated groups.

With NF/MBP-double immunofluoresent labeling, in sharp contrast to that of Empty Nerve Conduit (ENC) group (A-C), significant remyelination of regenerated axons was detected throughout the entire Nanofiber Nerve Conduit (NNC) 16 weeks after treatment (D-F). Representative transverse sections of proximal, middle and distal parts of Nerve Conduit (NC) were demonstrated at panel (A, D), (B, E), and (C, F) in ENC and NNC group, respectively. $(\mathrm{G})$ is the higher magnification of boxed area of $(\mathrm{E})$ with arrows indicating the representative remyelinated fibers. Furthermore, with electron microscopy, typical remyelinated fibers (arrowheads) could be found in both NC groups, while the diameter of the fiber and the thickness of the myelin are greater in NNC (H) compared with ENC (I). (J,K) showed the quantification analysis of the myelinated fiber caliber and the G-ratio ( an index of myelin thickness) separately. Both comparisons using student's t test indicated significantly statistical differences $\left({ }^{*} \mathrm{p}<0.05 ;{ }^{* *} \mathrm{p}<0.01\right)$. 

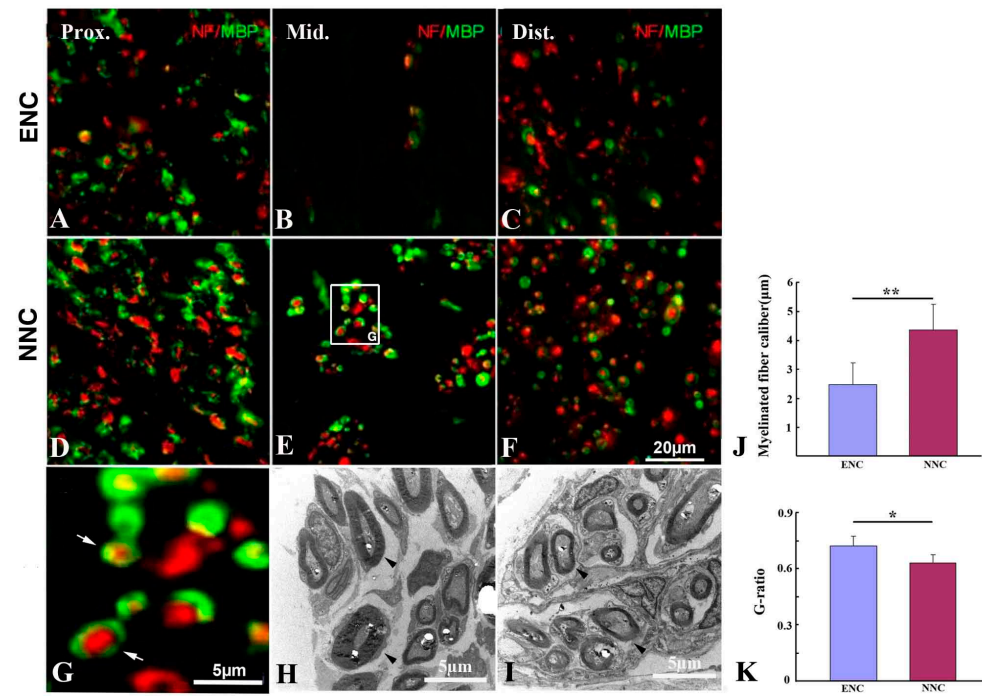

Figure 2. Remyelination of regenerated axons in the peripheral nerve conduits

\section{Author details}

Mingyong Gao ${ }^{1,7}$, Jiasong Guo ${ }^{5}$, Gilberto K. K. Leung ${ }^{6}$ and Wutian $\mathrm{Wu}^{1,2,3,4}$

1 Department of Anatomy, Li Ka Shing Faculty of Medicine, The University of Hong Kong, Hong Kong SAR, China

2 State Key Laboratory of Brain and Cognitive Sciences, China

3 Research Center of Reproduction, Development and Growth, Li Ka Shing Faculty of Medicine, The University of Hong Kong, Pokfulam, Hong Kong SAR, China

4 Joint Laboratory for Brain Function and Health (BFAH), Jinan University and The University of Hong Kong, Guangzhou, China

5 Department of Histology and Embryology, Southern Medical University, Guangzhou, China

6 Department of Surgery, Li Ka Shing Faculty of Medicine, The University of Hong Kong, Pokfulam, Hong Kong SAR, China

7 Department of Orthopeadics, Renmin hospital of Wuhan University, Wuhan, China 


\section{References}

[1] Battiston B, Raimondo S, Tos P, Gaidano V, Audisio C, Scevola A, Perroteau I, Geuna S. Tissue engineering of peripheral nerves. Int Rev Neurobiol. 2009; 87: 227-49.

[2] Chen VJ, Wei G, Ma PX, Nanostructured scaffolds for tissue engineering and regeneration, in: H.S. Nalwa (Ed.), Handbook of Nanostructured Biomaterials and Their Applications in Nanobiotechnology,vol. II, American Scientific Publishers, Stevenson Ranch, California, 2005; 415-35.

[3] Cullen DK, R Patel A, Doorish JF, Smith DH, Pfister BJ. Developing a tissue-engineered neural-electrical relay using encapsulated neuronal constructs on conducting polymer fibers. J Neural Eng. 2008;5(4):374-84.

[4] Cunha C, Panseri S, Antonini S.Emerging nanotechnology approaches in tissue engineering for peripheral nerve regeneration. Nanomedicine. 2011; 7(1):50-9.

[5] Dalton PD, Flynn L, Shoichet MS. Manufacture of poly(2-hydroxyethyl methacrylateco-methyl methacrylate)hydrogel tubes for use as nerve guidance channels. Biomaterials. 2002; 23(18):, 3843-51.

[6] Deumens R, Bozkurt A, Meek MF, Marcus MA, Joosten EA, Weis J, Brook GA. Repairing injured peripheral nerves: Bridging the gap. Prog Neurobiol. 2010; 92(3): 245-76.

[7] Ellis-Behnke RG, Liang YX, You SW, Tay DK, Zhang S, So KF, Schneider GE.. Nano neuro knitting: peptide nanofi ber scaffold for brain repair and axon regeneration with functional return of vision. Proc. Natl. Acad.Sci. USA. 2006; 103(13): 5054-9.

[8] Gelain F, Bottai D, Vescovi A, Zhang S. Designer self-assembling peptide nanofiber scaffolds for adult mouse neural stem cell 3-dimensional cultures. PLoS ONE. 2006; e119:1.

[9] Gelain F, Cigognini D, Caprini A, Silva D, Colleoni B, Donegá M, Antonini S, Cohen BE, Vescovi A.New bioactive motifs and their use in functionalized self-assembling peptides for NSC differentiation and neural tissue engineering. Nanoscale. 2012;4(9): 2946-57.

[10] Ghajar J. Traumatic brain injury. Lancet. 2000; 356(9233):923-9.

[11] Gordon T, Sulaiman O, Boyd JG., Experimental strategies to promote functional recovery after peripheral nerve injuries. J Peripher Nerv Syst. 2003; 8(4): 236-50.

[12] Guo J, Leung KKG, Su H, Yuan QJ, Wang L, Chu TH, Zhang W, Pu JKS, Ng GKP, Wong WM, Dai X, Wu WT.. Self-assembling peptide nanofi ber scaffold promotes the reconstruction of acutely injured brain.Nanomedicine. 2009; 5(3): 345-51. 
[13] Guo J, Su H, Zeng Y, Liang YX, Ellis-Behnke RG, So KF, Wu W. Reknitting the injured spinal cord by self-assembling peptide nanofi ber scaffold. Nanomedicine 2007; 3(4): 311-21.

[14] Jiang X, Mi R, Hoke A, Chew SY. Nanofibrous nerve conduit-enhanced peripheral nerve regeneration. J Tissue Eng Regen Med. 2012 Jun 15. [Epub ahead of print]

[15] Keeley RD, Nguyen KD, Stephanides MJ, Padilla J, Rosen JM. The artificial nerve graft: a comparison of blended elastomer-hydrogel with polyglycolic acid conduits. J Reconstr Microsurg 1991;7(2):93-100.

[16] Kehoe S, Zhang XF, Boyd D. FDA approved guidance conduits and wraps for peripheral nerve injury: a review of materials and efficacy. Injury. 2012; 43 (5):553-72.

[17] Kim H, Cooke MJ, Shoichet MS. Creating permissive microenvironments for stem cell transplantation into the central nervous system. Trends Biotechnol. 2012; 30(1): 55-63.

[18] Ma PX. Biomimetic materials for tissue engineering. Adv Drug Deliv Rev. 2008; 60(2): 184-98.

[19] Millesi H. Trauma involving the brachial plexus. In: Omer GE, Spinner M, Van Beek AL, eds. Management of Peripheral Nerve Problems. Philadelphia: Saunders; 1998;433-44.

[20] Noble J, Munro CA, Prasad VSSV, Midha R. Analysis of upper and lower extremity peripheral nerve injuries in a population of patients with multiple injuries. J Trauma. 1998; 45(1): 116-22.

[21] Panseri S, Cunha C, Lowery J, Del Carro U, Taraballi F, Amadio S, Vescovi A, Gelain F. Electrospun micro- and nanofiber tubes for functional nervous regeneration in sciatic nerve transections. BMC Biotechnol. 2008; 8:39.

[22] Prabhakaran MP, Venugopal JR, Chyan TT, Hai LB, Chan CK, Lim AY, Ramakrishna S. Electrospun biocomposite nanofibrous scaffolds for neural tissue engineering. Tissue Eng Part A. 2008;14(11):1787-97.

[23] Raimondo S, Fornaro M, Tos P, Battiston B, Giacobini-Robecchi MG, Geuna S. Perspectives in regeneration and tissue engineering of peripheral nerves. Ann Anat. 2011,193(4):334-40.

[24] Samadikuchaksaraei A. An overview of tissue engineering approaches for management of spinal cord injuries. J Neuroeng Rehabil. 2007; 4: 15.

[25] Toba T, Nakamura T, Lynn AK, Matsumoto K, Fukuda S, Yoshitani M, Hori Y, Shimizu Y. Evaluation of peripheral nerve regeneration across an 80-mm gap using a polyglycolic acid (PGA)--collagen nerve conduit filled with laminin-soaked collagen sponge in dogs. Int J Artif Organs. 2002; 25(3):230-7. 
[26] Taylor CA, Braza D, Rice JB, Dillingham T. The incidence of peripheral nerve injury in extremity trauma. Am J Phys Med Rehabil, 2008; 87(5): 381-85

[27] Tsai EC, Dalton PD, Shoichet MS, Tator CH. Matrix inclusion within synthetic hydrogel guidance channels improves specific supraspinal and local axonal regeneration after complete spinal cord transection. Biomaterials. 2006; 27(3):519-33.

[28] Tysseling-Mattiace VM, Sahni V, Niece KL, Birch D, Czeisler C, Fehlings MG, Stupp SI, Kessler JA.

[29] Self-assembling nanofibers inhibit glial scar formation and promote axon elongation after spinal cord injury. J Neurosci. 2008;28(13):3814-23.

[30] Walker PA, Aroom KR, Jimenez F, Shah SK, Harting MT, Gill BS, Cox CS Jr.. Advances in progenitor cell therapy using scaffolding constructs for central nervous system injury. Stem Cell Rev. 2009; 5(3): 283-300.

[31] Wang M, Zhai P, Chen X, Schreyer DJ, Sun X, Cui F. Bioengineered scaffolds for spinal cord repair. Tissue Eng Part B. 2011;17(3):177-94.

[32] Wang W, Itoh S, Matsuda A, Aizawa T, Demura M, Ichinose S, Shinomiya K, Tanaka J.Enhanced nerve regeneration through a bilayered chitosan tube: the effect of introduction of glycine spacer into the CYIGSR sequence. J Biomed Mater Res A. 2008; 85(4): 919-28.

[33] Xie J, MacEwan MR, Schwartz AG, Xia Y. Electrospun nanofibers for neural tissue engineering. Nanoscale. 2010; 2(1):35-44.

[34] Yang F, Murugan R, Ramakrishna S, Wang X, Ma YX, Wang S. Fabrication of nanostructured porous PLLA scaffold intended for nerve tissue engineering. Biomaterials 2004; 25(10):1891-900.

[35] Zhu Y, Wang A, Patel S, Kurpinski K, Diao E, Bao X, Kwong G, Young W, Li S. Engineering Bi-layer Nanofibrous Conduits for Peripheral Nerve Regeneration. Tissue Eng Part C Methods. 2011; 17(7):705-15. 
